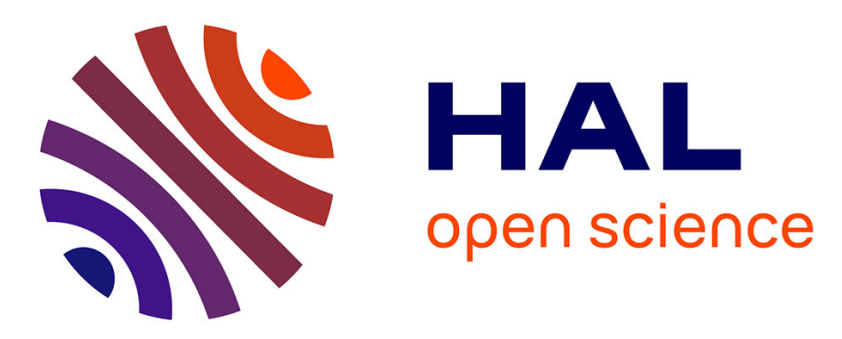

\title{
Supervisory Acceleration of Convergence for Homogeneous Systems
}

Denis Efimov, Arie Levant, Andrey Polyakov, Wilfrid Perruquetti

\section{To cite this version:}

Denis Efimov, Arie Levant, Andrey Polyakov, Wilfrid Perruquetti. Supervisory Acceleration of Convergence for Homogeneous Systems. International Journal of Control, 2017, 10.1080/00207179.2016.1269949 . hal-01411088

\section{HAL Id: hal-01411088 \\ https://hal.inria.fr/hal-01411088}

Submitted on 7 Dec 2016

HAL is a multi-disciplinary open access archive for the deposit and dissemination of scientific research documents, whether they are published or not. The documents may come from teaching and research institutions in France or abroad, or from public or private research centers.
L'archive ouverte pluridisciplinaire HAL, est destinée au dépôt et à la diffusion de documents scientifiques de niveau recherche, publiés ou non, émanant des établissements d'enseignement et de recherche français ou étrangers, des laboratoires publics ou privés. 


\title{
Supervisory Acceleration of Convergence for Homogeneous Systems
}

\author{
D. Efimov, A. Levant, A. Polyakov, W. Perruquetti
}

\begin{abstract}
The rate of convergence to the origin for a chain of integrators stabilized by homogeneous feedback is accelerated by a supervisory switching of control parameters. The proposed acceleration algorithm ensures a fixed-time convergence for otherwise exponentially or finite-time stable homogeneous closed-loop systems. Bounded disturbances are taken into account. The results are especially useful in the case of exponentially stable systems widespread in the practice. The proposed switching strategy is illustrated by computer simulation.
\end{abstract}

\section{INTRODUCTION}

State feedback stabilization of linear or nonlinear systems is a central problem in the control systems theory. There are many methods [3], [8], [11], which are differing by the requirements imposed on the plant model and on the guaranteed performance of the closed-loop system. By performance we mean: robustness with respect to external disturbances, measurement noises and small delays, rate and domain of convergence, overshooting, etc.

Convergence rate and time in closed-loop controlled systems are the main optimization criteria in applications. The present work studies the problem of adjusting the convergence rate for closed-loop homogeneous systems [20], [3]. It is a well-known fact that if a system is homogeneous with a negative/zero/positive degree and asymptotically stable, then actually it has a finite-time/exponential/asymptotic rate of convergence (in the case of positive degree the time of convergence to a sphere is uniformly bounded by a constant for any initial conditions, if the system is also locally finite-time converging, then it is called fixed-time stable) [?], [15], [16]. Despite of that, an additional problem of acceleration can be posed [2], [6], [7], i.e. the problem of changing of type of convergence (from asymptotic to finite-time or fixed-time, for example) by adjustment of parameters uniformly in bounded external disturbances.

In [2] for a scalar linear system with bounded perturbation a switching rule, which increases the scalar gain of linear feedback, has been proposed making the closed-loop system finite-time stable, an extension to planar mechanical systems is given in [1]. In [6], [7] for sliding mode control systems [19], [10], [14], [17] (which are frequently homogeneous systems with a negative degree) it has been proposed an algorithm of on-line switching between parameters, which ensures a desired accelerated rate of convergence for the closed-loop system. The proposed strategy consists in a proper decreasing the acceleration rate by parameter commutation till the normal one close to the origin. In the present work the following new extensions are proposed. First, the algorithms of fixed-time convergence are developed for exponentially converging systems with zero degree (linear systems). Second, the scenario with growing acceleration rate is also analyzed for a chain of integrators (in such a case the time when a control with augmented amplitude is applied can be seriously limited). Third, the algorithm of parameter switching for finite-time convergence to the origin is developed for homogeneous systems with zero and positive degree. Contrarily [9] the presence of external disturbances is taken into account, and the convergence rate is uniform with respect to bounded disturbances.

All authors are with Non-A team @ Inria, Parc Scientifique de la Haute Borne, 40 avenue Halley, 59650 Villeneuve d'Ascq, France and CRIStAL (UMR-CNRS 9189), Ecole Centrale de Lille, Cité Scientifique, 59651 Villeneuve-d'Ascq, France.

D. Efimov and A. Polyakov are with Department of Control Systems and Informatics, University ITMO, 49 av. Kronverkskiy, 197101 Saint Petersburg, Russia.

A. Levant is with School of Mathematical Sciences,Tel-Aviv University, Tel-Aviv 6997801, Israel.

This work was partially supported by ANR 15 CE23 0007 (Project Finite4SoS), the Government of Russian Federation (Grant 074-U01) and the Ministry of Education and Science of Russian Federation (Project 14.Z50.31.0031). 
The outline of this paper is as follows. Notation and preliminary results are introduced in sections II and III. The precise problem statement is given in Section IV. The proposed supervisory algorithms of switching among different sets of parameters ensuring a required acceleration are presented in Section V. All algorithms are illustrated by computer experiments with a benchmark example. Concluding remarks and discussion appear in Section VI.

\section{NOTATION}

Through the paper the following notation is used:

- $\mathbb{R}_{+}=\{x \in \mathbb{R}: x \geq 0\}$, where $\mathbb{R}$ is the set of real number.

- $|\cdot|$ denotes the absolute value in $\mathbb{R},\|\cdot\|$ denotes the Euclidean norm on $\mathbb{R}^{n},\|x\|_{\mathcal{A}}=\inf _{\xi \in \mathcal{A}}\|x-\xi\|$ is the distance from a point $x \in \mathbb{R}^{n}$ to a set $\mathcal{A} \subset \mathbb{R}^{n}$.

- For a (Lebesgue) measurable function $d: \mathbb{R}_{+} \rightarrow \mathbb{R}^{m}$ define the norm $\|d\|_{\left[t_{0}, t_{1}\right)}=\operatorname{ess}_{\sup } \operatorname{su}_{\left.t \in t_{0}, t_{1}\right)}\|d(t)\|$, then $\|d\|_{\infty}=$ $\|d\|_{[0,+\infty)}$ and the set of $d(t)$ with the property $\|d\|_{\infty}<+\infty$ we further denote as $\mathcal{L}_{\infty}$ (the set of essentially bounded measurable functions); $\mathcal{L}_{D}=\left\{d \in \mathcal{L}_{\infty}:\|d\|_{\infty} \leq D\right\}$ for any $D>0$.

- A continuous function $\alpha: \mathbb{R}_{+} \rightarrow \mathbb{R}_{+}$belongs to the class $\mathcal{K}$ if $\alpha(0)=0$ and the function is strictly increasing. The function $\alpha: \mathbb{R}_{+} \rightarrow \mathbb{R}_{+}$belongs to the class $\mathcal{K}_{\infty}$ if $\alpha \in \mathcal{K}$ and it is increasing to infinity. A continuous function $\beta: \mathbb{R}_{+} \times \mathbb{R}_{+} \rightarrow \mathbb{R}_{+}$belongs to the class $\mathcal{K} \mathcal{L}$ if $\beta(\cdot, t) \in \mathcal{K}_{\infty}$ for each fixed $t \in \mathbb{R}_{+}$and $\lim _{t \rightarrow+\infty} \beta(s, t)=0$ for each fixed $s \in \mathbb{R}_{+}$.

- A series of integers $1,2, \ldots, n$ is denoted by $\overline{1, n}$.

\section{Preliminaries}

Consider the following nonlinear system:

$$
\dot{x}(t)=f(x(t), d(t)), t \geq 0,
$$

where $x(t) \in \mathbb{R}^{n}$ is the state, $d(t) \in \mathbb{R}^{m}$ is the input, $d \in \mathcal{L}_{\infty} ; f: \mathbb{R}^{n+m} \rightarrow \mathbb{R}^{n}$ ensures forward existence of the system solutions at least locally, $f(0,0)=0$. For an initial condition $x_{0} \in \mathbb{R}^{n}$ and input $d \in \mathcal{L}_{\infty}$ define the corresponding solution by $X\left(t, x_{0}, d\right)$ for any $t \geq 0$ for which the solution exists.

Following [18], [11], [13], [16], let $\Omega$ be an open neighborhood of the origin in $\mathbb{R}^{n}$ and $D>0$.

Definition 1. At the steady state $x=0$ the system (1) for $d \in \mathcal{L}_{D}$ is said to be

(a) uniformly Lyapunov stable in $\Omega$ if for any $x_{0} \in \Omega$ and $d \in \mathcal{L}_{D}$ the solution $X\left(t, x_{0}, d\right)$ is defined for all $t \geq 0$, and for any $\epsilon>0$ there is $\delta>0$ such that for any $x_{0} \in \Omega$, if $\left\|x_{0}\right\| \leq \delta$ then $\left\|X\left(t, x_{0}, d\right)\right\| \leq \epsilon$ for all $t \geq 0$;

(b) uniformly asymptotically stable in $\Omega$ if it is uniformly Lyapunov stable in $\Omega$ and for any $\kappa>0$ and $\epsilon>0$ there exists $T(\kappa, \epsilon) \geq 0$ such that for any $x_{0} \in \Omega$ and $d \in \mathcal{L}_{D}$, if $\left\|x_{0}\right\| \leq \kappa$ then $\left\|X\left(t, x_{0}, d\right)\right\| \leq \epsilon$ for all $t \geq T(\kappa, \epsilon)$;

(c) uniformly finite-time stable in $\Omega$ if it is uniformly Lyapunov stable in $\Omega$ and uniformly finite-time converging in $\Omega$, i.e. for any $x_{0} \in \Omega$ and all $d \in \mathcal{L}_{D}$ there exists $0 \leq T<+\infty$ such that $X\left(t, x_{0}, d\right)=0$ for all $t \geq T$. The function $T_{0}\left(x_{0}\right)=\inf \left\{T \geq 0: X\left(t, x_{0}, d\right)=0 \forall t \geq T, \forall d \in \mathcal{L}_{D}\right\}$ is called the uniform settling time of the system (1);

(d) uniformly fixed-time stable in $\Omega$ if it is uniformly finite-time stable in $\Omega$ and $\sup _{x_{0} \in \Omega} T_{0}\left(x_{0}\right)<+\infty$.

The set $\Omega$ is called the domain of stability/attraction.

If $\Omega=\mathbb{R}^{n}$, then the corresponding properties are called global uniform Lyapunov/asymptotic/finite-time/fixed-time stability of (1) for $d \in \mathcal{L}_{D}$ at $x=0$.

Similarly stability notions can be defined with respect to a set, by replacing the distance to the origin in Definition 1 with the distance to an invariant set. For example, the global uniform finite-time stability with respect to a compact set $\mathcal{A} \subset \mathbb{R}^{n}$ is equivalent to the following two properties:

(i) global uniform Lyapunov stability: for any $x_{0} \in \mathbb{R}^{n}$ and $d \in \mathcal{L}_{D}$ the solution $X\left(t, x_{0}, d\right)$ is defined for all $t \geq 0$, and 
for any $\epsilon>0$ there is $\delta>0$ such that if $\left\|x_{0}\right\|_{\mathcal{A}} \leq \delta$ then $\left\|X\left(t, x_{0}, d\right)\right\|_{\mathcal{A}} \leq \epsilon$ for all $t \geq 0$;

(ii) global uniform finite-time convergence: for any $x_{0} \in \mathbb{R}^{n}$ and all $d \in \mathcal{L}_{D}$ there exists $0 \leq T<+\infty$ such that $\left\|X\left(t, x_{0}, d\right)\right\|_{\mathcal{A}}=0$ for all $t \geq T$.

\section{A. Weighted homogeneity}

Following [20], [3], [12], for strictly positive numbers $r_{i}, i=\overline{1, n}$ called weights and $\lambda>0$, one can define:

- the vector of weights $\mathbf{r}=\left(r_{1}, \ldots, r_{n}\right)^{T}, r_{\max }=\max _{1 \leq j \leq n} r_{j}$ and $r_{\min }=\min _{1 \leq j \leq n} r_{j}$;

- the dilation matrix function $\Lambda_{r}(\lambda)=\operatorname{diag}\left\{\lambda^{r_{i}}\right\}_{i=1}^{n}$, note that $\forall x \in \mathbb{R}^{n}$ and $\forall \lambda>0$ we have $\Lambda_{r}(\lambda) x=$ $\left(\lambda^{r_{1}} x_{1}, \ldots, \lambda^{r_{i}} x_{i}, \ldots, \lambda^{r_{n}} x_{n}\right)^{T}$

- the $\mathbf{r}$-homogeneous norm $\|x\|_{r}=\left(\sum_{i=1}^{n}\left|x_{i}\right|^{\frac{\rho}{r_{i}}}\right)^{\frac{1}{\rho}}$ for any $x \in \mathbb{R}^{n}$ and $\rho \geq r_{\max }$, then there exist $\underline{\sigma}, \bar{\sigma} \in \mathcal{K}_{\infty}{ }^{1}$ such that

$$
\underline{\sigma}\left(\|x\|_{r}\right) \leq\|x\| \leq \bar{\sigma}\left(\|x\|_{r}\right) \quad \forall x \in \mathbb{R}^{n}
$$

consequently, due to this equivalence of the norms $\|\cdot\|$ and $\|\cdot\|_{r}$, the homogeneous norm can be used in Definition 1.

- the unit sphere and the unit ball in the homogeneous norm $S_{r}=\left\{x \in \mathbb{R}^{n}:\|x\|_{r}=1\right\}$ and $B_{r}(\rho)=\left\{x \in \mathbb{R}^{n}:\|x\|_{r} \leq\right.$ $\rho\}$ for $\rho \geq 0$.

Definition 2. A function $g: \mathbb{R}^{n} \rightarrow \mathbb{R}$ is $\mathbf{r}$-homogeneous with degree $\mu \in \mathbb{R}$ if $\forall x \in \mathbb{R}^{n}$ and $\forall \lambda>0$ we have:

$$
\lambda^{-\mu} g\left(\Lambda_{r}(\lambda) x\right)=g(x)
$$

A vector field $\phi: \mathbb{R}^{n} \rightarrow \mathbb{R}^{n}$ is $\mathbf{r}$-homogeneous with degree $\nu \in \mathbb{R}$, with $\nu \geq-r_{\min }$ if $\forall x \in \mathbb{R}^{n}$ and $\forall \lambda>0$ we have:

$$
\lambda^{-\nu} \Lambda_{r}^{-1}(\lambda) \phi\left(\Lambda_{r}(\lambda) x\right)=\phi(x)
$$

which is equivalent to the $i$-th component of $\phi$ being a $\mathbf{r}$-homogeneous function of degree $r_{i}+\nu$.

The system (1) for $d=0$ is $\mathbf{r}$-homogeneous of degree $\nu$ if the vector field $f$ is $\mathbf{r}$-homogeneous of degree $\nu$ for $d=0$.

An important advantage of $\mathbf{r}$-homogeneous systems is that their rate of convergence can be evaluated qualitatively based on their degree.

Lemma 1. [3] If (1) for $d=0$ is $\mathbf{r}$-homogeneous of degree $\nu$ and asymptotically stable at the origin, then it is

(i) globally finite-time stable at the origin if $\nu<0$;

(ii) globally exponentially stable at the origin if $\nu=0$;

(iii) globally fixed-time stable with respect to the unit ball $B_{r}(1)$ if $\nu>0$.

For $\mathbf{r}$-homogeneous system (1) with degree $\nu$ and $d=0$, the solutions also admit a kind of homogeneity as functions of time $\forall x_{0} \in \mathbb{R}^{n}$ and $\forall t \in \mathbb{R}$ :

$$
X\left(t, \Lambda_{r}(\lambda) x_{0}, 0\right)=\Lambda_{r}(\lambda) X\left(\lambda^{\nu} t, x_{0}, 0\right) \quad \forall \lambda>0
$$

Definition 3. For $q>1$ define $T_{q}: \mathbb{R}^{n} \rightarrow \mathbb{R}_{+}$such that

$$
\left\|X\left(T_{q}\left(x_{0}\right), x_{0}, 0\right)\right\|_{r}=q^{-1}\left\|x_{0}\right\|_{r}
$$

i.e. it is the function of contraction in $q$ times.

${ }^{1}$ For example,

$$
\underline{\sigma}(s)=\left\{\begin{array}{ll}
n^{-\frac{r_{\max }}{\rho}} s^{r_{\max }} & \text { if } s \leq n^{\frac{1}{\rho}} \\
n^{-\frac{r_{\min }}{\rho}} s^{r_{\text {min }}} & \text { if } s>n^{\frac{1}{\rho}}
\end{array}, \bar{\sigma}(s)=\sqrt{n}\left\{\begin{array}{ll}
s^{r_{\min }} & \text { if } s \leq 1 \\
s^{r_{\max }} & \text { if } s>1
\end{array} .\right.\right.
$$


From the properties stated above it is easy to show that $T_{q}$ is $\mathbf{r}$-homogeneous with degree $-\nu$ :

$$
T_{q}\left(\Lambda_{r}(\lambda) x_{0}\right)=\lambda^{-\nu} T_{q}\left(x_{0}\right) \quad \forall x_{0} \in \mathbb{R}^{n} \forall \lambda>0 .
$$

\section{PRoblem introduction}

In this section the system of interest is introduced with a basic control, next some their properties used in the sequel are discussed, and finally the problem statement is given.

\section{A. Basic system and its properties}

Consider a chain of integrators stabilized in finite-time or exponentially by a state feedback $K: \mathbb{R}^{n} \rightarrow \mathbb{R}$ :

$$
\dot{x}(t)=f(x(t), d(t))=A x(t)+b[K(x(t))+d(t)], t \geq 0,
$$

where $x(t) \in \mathbb{R}^{n}$ is the state vector, $d(t) \in \mathbb{R}$ is the exogenous disturbance, $d \in \mathcal{L}_{D}$ for some $D>0$ (i.e. $\|d\|_{\infty} \leq D$ ); the matrices

$$
A=\left[\begin{array}{ccccc}
0 & 1 & 0 \ldots 0 & 0 \\
0 & 0 & 1 \ldots 0 & 0 \\
\vdots & \vdots & \ddots & \vdots \\
0 & 0 & 0 \ldots 0 & 1 \\
0 & 0 & 0 \ldots 0 & 0
\end{array}\right], b=\left[\begin{array}{c}
0 \\
\vdots \\
0 \\
1
\end{array}\right]
$$

are in the canonical form. For $r_{i}=1+(i-1) \nu$ with $i=\overline{1, n}$ and $\nu>-n^{-1}$ the function $K$ is $\mathbf{r}$-homogeneous of degree $1+n \nu$, i.e.

$$
K\left(\Lambda_{r}(\lambda) x\right)=\lambda^{1+n \nu} K(x) \quad \forall x \in \mathbb{R}^{n} \forall \lambda>0
$$

then the system (2) is $\mathbf{r}$-homogeneous of degree $\nu$ for $d=0$ :

$$
f\left(\Lambda_{r}(\lambda) x, 0\right)=\lambda^{\nu} \Lambda_{r}(\lambda) f(x, 0) \quad \forall x \in \mathbb{R}^{n} \forall \lambda>0 .
$$

Various examples of such a control law $K(x)$ can be proposed: linear with $\nu=0$ [11] or nonlinear for $\nu \neq 0$ [5].

Remark 1. The case $\nu=-n^{-1}$ leads to a discontinuous control application (function $K$ is $\mathbf{r}$-homogeneous of degree 0 in this case) and is not considered here since it was investigated in [6], [7].

Assumption 1. The system (2) is globally asymptotically stable for $d=0$.

If Assumption 1 is satisfied, then convergence to the origin takes place in finite-time (for $\nu<0$ ) or exponentially (for $\nu=0$, see Lemma 1).

Claim 1. Let Assumption 1 be satisfied and $\nu>-n^{-1}$, then the system (2) is input-to-state stable with respect to the input $d$, i.e. for any $x_{0} \in \mathbb{R}^{n}$ and $d \in \mathcal{L}_{\infty}$ there exist $a_{1} \geq 1, a_{2}>0$ and $\gamma \in \mathcal{K}_{\infty}$ such that

$$
\left\|X\left(t, x_{0}, d\right)\right\|_{r} \leq \beta\left(\left\|x_{0}\right\|_{r}, t\right)+\gamma\left(\|d\|_{\infty}\right) \quad \forall t \geq 0
$$

where

$$
\beta(s, t)=\left\{\begin{array}{lll}
\frac{s}{\left[a_{1}+a_{2} \nu s^{\nu} t\right]^{1 / \nu}} & \nu>0 \\
\left\{\left[a_{1} s^{-\nu}+a_{2} \nu t\right]^{-1 / \nu}\right. & t<-\frac{a_{1} s^{-\nu}}{a_{2} \nu} & \\
0 & t \geq-\frac{a_{1} s^{-\nu}}{a_{2} \nu} & \\
0 & & \nu=0
\end{array}\right.
$$


is a function from class $\mathcal{K} \mathcal{L}$.

Proof. According to Theorem 7 of [4], under Assumption 1 the system (2) is input-to-state stable with respect to the input $d$ if $\nu+r_{\min }>0\left(\vartheta_{\max }=0\right.$ in this case, see [4]). If $\nu>0$ then the last inequality takes the form $\nu+1>0$ and it is trivially satisfied, if $\nu<0$ then $\nu+r_{\min }=\nu+1+(n-1) \nu>0$ is satisfied for all $\nu>-n^{-1}$, then in both cases there exists some $\beta \in \mathcal{K} \mathcal{L}$. The particular structure of $\beta$ follows homogeneity of the system and it is also a consequence of result obtained in the proof of Theorem 7 of [4] (the estimate for Lyapunov function $V$ ).

An expression for $\gamma$ can also be found in [4], Corollary 3.

For $d=0$, calculating an estimate on the function of contraction in $q \geq a_{1} \geq 1$ times (see Definition 3) from (3) we obtain:

$$
T_{q}\left(x_{0}\right)=\left\{\begin{array}{ll}
\frac{q^{\nu}-a_{1}}{a_{2} \nu}\left\|x_{0}\right\|_{r}^{-\nu} & \nu \neq 0 \\
a_{2}^{-1} \ln \left(a_{1} q\right) & \nu=0
\end{array},\right.
$$

which is $\mathbf{r}$-homogeneous with degree $-\nu$ as it was mentioned.

For the case of non-zero inputs $d$ the following counterpart of $T_{q}\left(x_{0}\right)$ can be introduced. For given $q \geq a_{1} \geq 1$ and $w \in(0,1)$ the relative function of contraction in $q$ times $T_{q, w}^{\prime}: \mathbb{R}^{n} \rightarrow \mathbb{R}_{+}$characterizes the time of state amplitude contraction in $q$ times under assumption that $\gamma\left(\|d\|_{\infty}\right) \leq \frac{w}{q}\left\|x_{0}\right\|_{r}$, i.e. $\left\|X\left(T_{q, w}^{\prime}\left(x_{0}\right), x_{0}, d\right)\right\|_{r} \leq q^{-1}\left\|x_{0}\right\|_{r}$ for a given $x_{0} \in \mathbb{R}^{n}$ and for any $d \in \mathcal{L}_{\infty}$ such that $\|d\|_{\infty} \leq \gamma^{-1}\left(\frac{w}{q}\left\|x_{0}\right\|_{r}\right)$. From (3) it has an estimate:

$$
T_{q, w}^{\prime}\left(x_{0}\right)=\left\{\begin{array}{ll}
\frac{\left(\frac{q}{1-w}\right)^{\nu}-a_{1}}{a_{2} \nu}\left\|x_{0}\right\|_{r}^{-\nu} & \nu \neq 0 \\
a_{2}^{-1} \ln \left(\frac{a_{1} q}{1-w}\right) & \nu=0
\end{array} .\right.
$$

As we see, the relative function $T_{q, w}^{\prime}$ is also $\mathbf{r}$-homogeneous with degree $-\nu$. Of course, if $\gamma\left(\|d\|_{\infty}\right)>\frac{1}{q}\left\|x_{0}\right\|_{r}$, then convergence of the system solution in $q$ times cannot be guaranteed.

\section{B. The problem statement}

The problem studied in this work: is it possible to ensure the uniform fixed-time stabilization in (2) to a fixed ball by switching among different sets of coefficients in the feedback $K$ for $d \in \mathcal{L}_{D}$ with a given $D>0$. The radius of the ball can be taken zero, provided the system homogeneity degree is non-zero. Such a problem has been already considered in [1], [2] (finite-time case) and in [6], [7] for the sliding mode case with $\nu=-n^{-1}$, and following that works, instead of (2) we will consider for all integer $i \geq 0$ the system:

$$
\begin{gathered}
\dot{z}(t)=A z(t)+b\left[u\left(\mu_{i}, z(t)\right)+d(t)\right], t \in\left[t_{i}, t_{i+1}\right), \\
u\left(\mu_{i}, z\right)=\mu_{i}^{n} K\left(M_{i} z\right)
\end{gathered}
$$

where $z(t) \in \mathbb{R}^{n}$ is the state vector, $u: \mathbb{R}^{n+1} \rightarrow \mathbb{R}$ is the control, $d(t) \in \mathbb{R}$ is the disturbance as previously, $d \in \mathcal{L}_{D} ; A$, $b$ and $K$ are as before; $M_{i}=\operatorname{diag}\left\{\mu_{i}^{1-k}\right\}_{k=1}^{n}$ and $\mu_{i} \geq 1$ is a sequence of parameters, which stay constant on the interval $\left[t_{i}, t_{i+1}\right)$ and change their values at instants $t_{i}, i \geq 0\left(t_{0}=0\right)$. It is required to determine the instants $t_{i}, i \geq 0$ and the discrete-time update law for $\mu_{i}$ such that (4) becomes uniformly fixed-time stable with respect to $B_{r}(1)$. Obviously, (4) is $\mathbf{r}$-homogeneous for $d=0$ of degree $\nu$ for any $i \geq 0$.

Similarly, a uniform finite-time stabilization problem can be studied for $\nu \geq 0$.

\section{SUPERVISORY ALGORITHM DESIGN}

Let us introduce a dynamical system for $t \in\left[t_{i}, t_{i+1}\right)$ :

$$
\begin{aligned}
\dot{x}(t) & =\mu_{i} f\left(x(t), \mu_{i}^{-n} d(t)\right) \\
& =\mu_{i}\left\{A x(t)+b\left[K(x(t))+\mu_{i}^{-n} d(t)\right]\right\},
\end{aligned}
$$


where $\mu_{i}$ is the same as in (4), and after update of $\mu_{i}$ to $\mu_{i+1}$ at the instant of time $t_{i+1}$ we have a state resetting for $x(t)$ :

$$
x\left(t_{i+1}\right)=M_{i+1} M_{i}^{-1} x\left(t_{i+1}^{-}\right),
$$

where $x\left(t_{i+1}^{-}\right)$denotes the left limit of $x(t)$ as $t$ is approaching $t_{i+1}$ from the left. As we can conclude, (5), (6) is a hybrid system, which has to be augmented by rules for assignment of switching instants $t_{i}$ and for update of $\mu_{i}$, for all $i \geq 0$.

Obviously, $z(t)=M_{i}^{-1} x(t)$ for $t \in\left[t_{i}, t_{i+1}\right)$ is the corresponding solution of (4). Therefore, in order to design the supervisory algorithms for selection of $t_{i}$ and $\mu_{i}$ we will consider below the hybrid system (5), (6).

Claim 2. Let Assumption 1 be satisfied and $\nu>-n^{-1}$. For any $D>0$ there is $\mu_{D}>\max \left\{1, \frac{D}{\gamma^{-1}(1)}\right\}^{1 / n}$ such that for any fixed $\mu_{i} \geq \mu_{D}$ the system (5) is globally uniformly finite-time stable with respect to the unit ball $B_{r}(1)$.

Proof. It is straightforward to verify that for any fixed $\mu_{i}$ the system (5) is $\mathbf{r}$-homogeneous of degree $\nu$ and globally asymptotically stable for $d=0$. Since all conditions of Claim 1 are satisfied, the system (5) is input-to-state stable with respect to the input $d$, and due to relations between (2) and (5) we have that for all $x_{0} \in \mathbb{R}^{n}, d \in \mathcal{L}_{D}$ and $t \geq 0$ :

$$
\begin{aligned}
\left\|X_{\mu}\left(t, x_{0}, d\right)\right\|_{r} & \leq \beta\left(\left\|x_{0}\right\|_{r}, \mu_{i} t\right)+\gamma\left(\left\|\mu_{i}^{-n} d\right\|_{\infty}\right) \\
& \leq \beta\left(\left\|x_{0}\right\|_{r}, \mu_{i} t\right)+\gamma\left(\mu_{i}^{-n}\|d\|_{\infty}\right) \\
& \leq \beta\left(\left\|x_{0}\right\|_{r}, \mu_{i} t\right)+\gamma\left(\mu_{i}^{-n} D\right)
\end{aligned}
$$

provided that $\mu_{i}>1$, where $X_{\mu}\left(t, x_{0}, d\right)$ is the corresponding solution of (5). Then due to properties of functions from class $\mathcal{K} \mathcal{L}$ there exists $\mu_{D}>\max \left\{1, \frac{D}{\gamma^{-1}(1)}\right\}^{1 / n}$ such that $\gamma\left(\mu_{i}^{-n} D\right)<1$ for any fixed $\mu_{i} \geq \mu_{D}$ and the system (5) is globally uniformly finite-time stable with respect to the unit ball $B_{r}(1)$.

Thus, the system can be made finite-time stable with respect to the unit ball $B_{r}(1)$ by a single choice of $\mu_{i}$, and our goal is to accelerate the system by switching among different values of $\mu_{i}$ in order to make the system uniformly fixed-time stable with respect to $B_{r}(1)$.

\section{A. First strategy for $-n^{-1}<\nu<0$}

In this work the following algorithm is proposed for all $i \geq 0$ :

$$
t_{i+1}=\min _{t \geq t_{i}:\left\|M_{i} z(t)\right\|_{r} \leq q^{-1-i}\|z(0)\|_{r}} t,
$$

where $q>a_{1} \geq 1$ is a tuning parameter, define $i^{*}=\operatorname{ceil}\left[\max \left\{0, \ln _{q}\|z(0)\|_{r}\right\}\right]$ (the function ceil $[s]$ provides the smallest integer bigger than $s$ ) and

$$
\mu_{i}=\mu_{D}\left\{\begin{array}{ll}
q^{-i(\alpha-\nu)}\|z(0)\|_{r}^{-\nu} & \text { if } i<i^{*} \\
1 & \text { otherwise }
\end{array},\right.
$$

where $\alpha \in(\nu, 0)$ is another tuning parameter and $\mu_{D}>0$ comes from Claim 2. It is necessary to show that the solutions of (5), (6) are well defined for the supervisory algorithm (7), (8), and that the closed loop system converges to $B_{r}(1)$ in a fixed time.

Note that for each constant $\mu_{i}$ the system (5) is converging to $B_{r}(1)$ (see the result of Claim 2), then always there exists an instant $t_{i+1}$ defined by (7) for all $i=\overline{0, i^{*}}$. Since $\alpha-\nu>0$, from (8) we obtain that $\mu_{i}>\mu_{D}$ for all $i=\overline{0, i^{*}-1}$ and $\mu_{i}=\mu_{D}$ for all $i \geq i^{*}$. Therefore, for all $t \geq t_{i^{*}}$ the system (5), (6), (7), (8) is reduced to (2), which is finite-time stable with respect to $B_{r}(1)$, and $\left\|x\left(t_{i^{*}}\right)\right\|_{r} \leq 1$. The solutions of this system are well defined for all $t \geq t_{i^{*}}$ (there is no more 
jump in the state), and it is necessary to consider what happens for $t \in\left[0, t_{i^{*}}\right)$ and estimate the time of reaching the unit ball $t_{i^{*}}$. To this end, from (8) we obtain for $i=\overline{0, i^{*}-1}$ :

$$
\begin{gathered}
M_{i+1} M_{i}^{-1}=\operatorname{diag}\left\{\left(\frac{\mu_{i+1}}{\mu_{i}}\right)^{1-k}\right\}_{k=1}^{n} \\
=\operatorname{diag}\left\{q^{(\alpha-\nu)(k-1)}\right\}_{k=1}^{n},
\end{gathered}
$$

and $M_{i+1} M_{i}^{-1}$ is stretching since $q>1$ and $\alpha-\nu>0$, i.e.

$$
\begin{aligned}
\left\|x\left(t_{i+1}\right)\right\|_{r} & <\left\|x\left(t_{i+1}^{+}\right)\right\|_{r} \leq q^{\frac{(\alpha-\nu)(n-1)}{1+\nu(n-1)}}\left\|x\left(t_{i+1}\right)\right\|_{r} \\
& \leq q^{\frac{(\alpha-\nu)(n-1)}{1+\nu(n-1)}-1-i}\|z(0)\|_{r}
\end{aligned}
$$

where the last step is obtained from (7). This property also implies that $t_{i+1}-t_{i}>0$ and the solutions of the system (5), (6), (7), (8) are well defined.

The instant $t_{i+1}$ will be generated by (7) when $\left\|x\left(t_{i+1}\right)\right\|_{r}=q^{-1-i}\|z(0)\|_{r}$ and on the interval of time $\left[t_{i}, t_{i+1}\right)$ the dynamics of $x(t)$ is governed by (5) for $\mu_{i}=\mu_{D} q^{-i(\alpha-\nu)}\|z(0)\|_{r}^{-\nu}$. On this interval $\left[t_{i}, t_{i+1}\right)$ the state $x(t)$ has to decrease in $\rho=q^{\frac{1+\alpha(n-1)}{1+\nu(n-1)}}$ times, i.e. the norm $\|x(t)\|_{r}$ goes down from the level $q^{\frac{(\alpha-\nu)(n-1)}{1+\nu(n-1)}-i}\|z(0)\|_{r}$ till $q^{-1-i}\|z(0)\|_{r}$. Let $T_{\rho, w}^{\prime}: \mathbb{R}^{n} \rightarrow \mathbb{R}_{+}$be the relative function of contraction in $\rho>1$ times with some $w \in(0,1)$ for $(2)$. For $i=\overline{0, i^{*}}$ in $(7)$ we have that $\left\|x\left(t_{i}\right)\right\|_{r} \geq q^{-1}$ and if $\mu_{D}>\max \left\{1, \frac{D}{\gamma^{-1}\left(q^{-1}\right)}\right\}$, then $T_{\rho, w}^{\prime}\left(x\left(t_{i}\right)\right)$ can be used for all $i=\overline{0, i^{*}-1}$ in order to evaluate the length of the interval $\left[t_{i}, t_{i+1}\right)$, therefore

$$
\begin{aligned}
t_{i+1}-t_{i} & =\mu_{i}^{-1} T_{\rho, w}^{\prime}\left(x\left(t_{i}\right)\right) \\
& \leq \mu_{i}^{-1} q^{\left(i-\frac{(\alpha-\nu)(n-1)}{1+\nu(n-1)}\right) \nu}\|z(0)\|_{r}^{-\nu} T_{\rho, w}^{\prime}(\xi) \\
& \leq \mu_{D}^{-1} q^{\alpha i-\frac{(\alpha-\nu)(n-1)}{1+\nu(n-1)} \nu} T_{\rho, w}^{\prime}(\xi)
\end{aligned}
$$

where $z(0)=\Lambda_{r}(\lambda) \xi$ for some $\xi$ from the unit sphere $S_{r}=\left\{\xi \in \mathbb{R}^{n}:\|\xi\|_{r}=1\right\}$. Let $\bar{T}_{\rho, w}^{\prime}=\sup _{\|\xi\|_{r}=1} T_{\rho, w}^{\prime}(\xi)$, then

$$
\begin{aligned}
t_{i^{*}} & =\sum_{i=0}^{i^{*}-1}\left(t_{i+1}-t_{i}\right) \\
& \leq \mu_{D}^{-1} \sum_{i=0}^{i^{*}-1} q^{\alpha i-\frac{(\alpha-\nu)(n-1)}{1+\nu(n-1)} \nu} \bar{T}_{\rho, w}^{\prime} \\
& =\mu_{D}^{-1} q^{-\frac{(\alpha-\nu)(n-1)}{1+\nu(n-1)} \nu} \bar{T}_{\rho, w}^{\prime} \sum_{i=0}^{i^{*}-1} q^{\alpha i} \\
& \leq \mu_{D}^{-1} \frac{q^{-\frac{(\alpha-\nu)(n-1)}{1+\nu(n-1)} \nu}}{1-q^{\alpha}} \bar{T}_{\rho, w}^{\prime},
\end{aligned}
$$

where the fact that $q^{\alpha}<1$ has been used on the last step. Therefore, the time of convergence to the unit ball $t_{i *}$ in the system (5), (6), (7), (8) is finite and independent in initial conditions, as it was necessary to show. The following result has been proven.

Theorem 1. Let for the system (4) with $d \in \mathcal{L}_{D}$ and $\nu \in\left(-n^{-1}, 0\right)$ the supervisory algorithm be selected as in (7), (8) with $q>a_{1}, \mu_{D}>\max \left\{1, \frac{D}{\gamma^{-1}\left(q^{-1}\right)}\right\}^{1 / n}$ and $\nu<\alpha<0$, then the solutions of the closed-loop system are well defined for all $t \geq 0$ and it is globally uniformly fixed-time stable with respect to $B_{r}(1)$ with the settling time bounded by

$$
\mu_{D}^{-1} \frac{q^{-\frac{(\alpha-\nu)(n-1)}{1+\nu(n-1)} \nu}}{1-q^{\alpha}} \bar{T}_{\rho, w}^{\prime},
$$

where $\bar{T}_{\rho, w}^{\prime}=\sup _{\|\xi\|_{r}=1} T_{\rho, w}^{\prime}(\xi)$ with $\rho=q^{\frac{1+\alpha(n-1)}{1+\nu(n-1)}}$ and $w \in(0,1)$ for the system (2). 

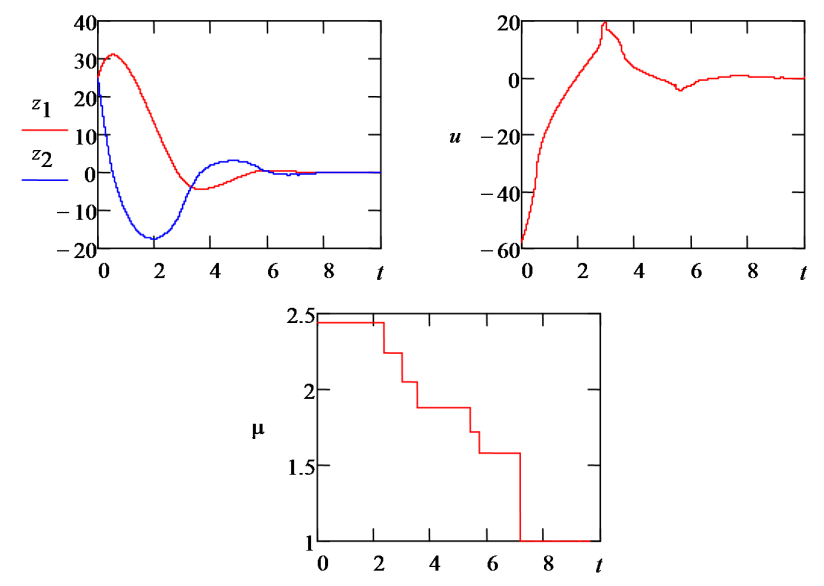

Figure 1. Fixed-time stabilization for $\nu<0$ with decreasing $\mu_{i}$

Remark 2. The same result can be obtained substituting (8) with

$$
\mu_{i}=\mu_{D} \begin{cases}q^{i(\alpha-\nu)}\|z(0)\|_{r}^{-\nu} & \text { if } i<i^{*} \\ 1 & \text { otherwise }\end{cases}
$$

for $\alpha \in(2 \nu, \nu)$.

Remark 3. A straightforward analysis shows that the function

$$
\sigma(q)=\frac{q^{-\frac{(\alpha-\nu)(n-1)}{1+\nu(n-1)} \nu}}{1-q^{\alpha}}
$$

has its minimum for

$$
q^{*}=\left(\frac{\nu(\alpha-\nu)(n-1)}{\alpha+\nu(2 \alpha-\nu)(n-1)}\right)^{1 / \alpha}
$$

providing the optimal selection of $q>1$ ensuring the fastest convergence of (4), (7), (8).

Example 1. Let us consider (2) with $n=2$ and the state feedback

$$
K(x)=-\left|x_{1}\right|^{1+2 \nu} \operatorname{sign}\left(x_{1}\right)-\left|x_{2}\right|^{\frac{1+2 \nu}{1+\nu}} \operatorname{sign}\left(x_{2}\right),
$$

borrowed from [5], which satisfies all required conditions. Select $D=1$ and

$$
d(t)=D \sin (15 t)
$$

for simulation, let $\nu=-0.25, q=2, \mu_{D}=1$ and $\alpha=-0.125$. An example of the system trajectories with the supervisory algorithm (7), (8) is given in Fig. 1.

B. Second strategy for $-n^{-1}<\nu \leq 0$

The algorithm for generation of instants $t_{i}$ will be selected as previously in (7), and

$$
\mu_{i}=\mu_{D}\left\{\begin{array}{ll}
q^{i \alpha}\|z(0)\|_{r}^{\theta} & \text { if } i<i^{*} \\
1 & \text { otherwise }
\end{array},\right.
$$

where $\alpha \in \mathbb{R}$ and $\theta \in \mathbb{R}$ are tuning parameters, $\mu_{D}$ is selected in accordance with Claim 2. Again, it is necessary to show that the solutions of (5), (6) are well defined for the supervisory algorithm (7), (9), and that the closed loop system converges to $B_{r}(1)$ in a fixed time. 
For all $t \geq t_{i^{*}}$ the system (5), (6), (7), (9) is reduced to (2), which is uniformly finite-time stable with respect to $B_{r}(1)$, and $\left\|x\left(t_{i^{*}}\right)\right\|_{r} \leq 1$. The solutions of this system are well defined for all $t \geq t_{i^{*}}$, and it is necessary to consider the system behavior for $t \in\left[0, t_{i^{*}}\right)$ and estimate the time $t_{i^{*}}$ of convergence to the unit ball $B_{r}(1)$. From (9) we obtain for all $i=\overline{0, i^{*}-1}$ :

$$
\begin{gathered}
M_{i+1} M_{i}^{-1}=\operatorname{diag}\left\{\left(\frac{\mu_{i+1}}{\mu_{i}}\right)^{1-k}\right\}_{k=1}^{n} \\
=\operatorname{diag}\left\{q^{\alpha(1-k)}\right\}_{k=1}^{n},
\end{gathered}
$$

and $M_{i+1} M_{i}^{-1}$ is stretching only if $\alpha<0$ (since $q>1$ ):

$$
\begin{aligned}
\left\|x\left(t_{i+1}^{+}\right)\right\|_{r} & \leq q^{\varrho(\alpha)}\left\|x\left(t_{i+1}\right)\right\|_{r} \\
& =q^{\varrho(\alpha)-1-i}\|z(0)\|_{r}
\end{aligned}
$$

with

$$
\varrho(\alpha)=\left\{\begin{array}{ll}
\frac{-\alpha(n-1)}{1+(n-1) \nu} & \text { if } \alpha<0 \\
0 & \text { otherwise }
\end{array} .\right.
$$

By the same arguments

$$
\begin{aligned}
\left\|x\left(t_{i+1}^{+}\right)\right\|_{r} & \geq q^{\chi(\alpha)}\left\|x\left(t_{i+1}\right)\right\|_{r} \\
& =q^{\chi(\alpha)-1-i}\|z(0)\|_{r}
\end{aligned}
$$

where

$$
\chi(\alpha)=\left\{\begin{array}{ll}
\frac{-\alpha(n-1)}{1+(n-1) \nu} & \text { if } \alpha>0 \\
0 & \text { otherwise }
\end{array},\right.
$$

The instant $t_{i+2}$ is well defined in (7) if $\chi(\alpha)>-1$. From the expression of $\chi(\alpha)$ this inequality is satisfied if $-\frac{1}{n-1}-\nu<-\alpha$ for $\alpha>0$, or equivalently $0<\alpha<\frac{1}{n-1}+\nu$. Since $\nu \in\left(-\frac{1}{n}, 0\right]$, then the interval $\left(0, \frac{1}{n-1}+\nu\right)$ is always non empty.

The instant $t_{i+1}$ will be generated by (7) when $\left\|x\left(t_{i+1}\right)\right\|_{r}=q^{-1-i}\|z(0)\|_{r}$ and on the interval of time $\left[t_{i}, t_{i+1}\right)$ the dynamics of $x(t)$ is governed by (5) for $\mu_{i}=\mu_{D} q^{i \alpha}\|z(0)\|_{r}^{\theta}$. On this interval $\left[t_{i}, t_{i+1}\right)$ the state $x(t)$ has to decrease in

$$
\rho=q^{1+\varrho(\alpha)}
$$

times. Let $T_{\rho, w}^{\prime}: \mathbb{R}^{n} \rightarrow \mathbb{R}_{+}$be the relative function of contraction in $\rho>1$ times with some $w \in(0,1)$ for (2), if $\mu_{D}>\max \left\{1, \frac{D}{\gamma^{-1}\left(q^{-1}\right)}\right\}^{1 / n}$, then $T_{\rho, w}^{\prime}\left(x\left(t_{i}\right)\right)$ can be used for all $i=\overline{0, i^{*}-1}$ in order to evaluate the length of the interval $\left[t_{i}, t_{i+1}\right)$ and

$$
\begin{gathered}
t_{i+1}-t_{i}=\mu_{i}^{-1} T_{\rho, w}^{\prime}\left(x\left(t_{i}\right)\right) \\
\leq \mu_{D}^{-1} q^{i(\nu-\alpha)-\nu \varrho(\alpha)}\|z(0)\|_{r}^{-\theta-\nu} T_{\rho}(\xi)
\end{gathered}
$$

where $z(0)=\Lambda_{r}(\lambda) \xi$ for some $\xi$ from the unit sphere $S_{r}=\left\{\xi \in \mathbb{R}^{n}:\|\xi\|_{r}=1\right\}$ as before. From the expressions above, the interval length $t_{i+1}-t_{i}$ is decreasing with $i$ and independent in $\|z(0)\|_{r}$ if

$$
\alpha>\nu,-\nu \leq \theta .
$$


Indeed, in this case $\|z(0)\|_{r}^{-\theta-\nu} \leq 1$ for all $\|z(0)\|_{r} \geq 1$. Let $\bar{T}_{\rho, w}^{\prime}=\sup _{\|\xi\|_{r}=1} T_{\rho, w}^{\prime}(\xi)$, then

$$
\begin{aligned}
t_{i^{*}} & =\sum_{i=0}^{i^{*}-1}\left(t_{i+1}-t_{i}\right) \\
& \leq \mu_{D}^{-1} \sum_{i=0}^{i^{*}-1} q^{i(\nu-\alpha)-\nu \varrho(\alpha)} \bar{T}_{\rho, w}^{\prime} \\
& =\mu_{D}^{-1} q^{-\nu \varrho(\alpha)} \bar{T}_{\rho, w}^{\prime} \sum_{i=0}^{i^{*}-1} q^{i(\nu-\alpha)} \\
& \leq \mu_{D}^{-1} \frac{q^{-\nu \varrho(\alpha)}}{1-q^{\nu-\alpha}} \bar{T}_{\rho, w}^{\prime},
\end{aligned}
$$

where the fact that $q^{\nu-\alpha}<1$ has been used on the last step. Therefore, the time of convergence to the unit ball $t_{i^{*}}$ in the system (5), (6), (7), (9) is finite and independent in initial conditions, as it was necessary to show. The following result has been proven.

Theorem 2. Let for the system (4) with $\nu \in\left(-\frac{1}{n}, 0\right]$ and $d \in \mathcal{L}_{D}$ the supervisory algorithm be selected as in (7), (9) with $q>a_{1}, \mu_{D}>\max \left\{1, \frac{D}{\gamma^{-1}\left(q^{-1}\right)}\right\}^{1 / n}, \alpha \in\left(\nu, \frac{1}{n-1}+\nu\right)$ and $-\nu \leq \theta$, then the solutions of the closed-loop system are well defined for all $t \geq 0$ and for any $z(0) \in \mathbb{R}^{n}$ the uniform time of convergence to the unit ball $B_{r}(1)$ is less than

$$
\mu_{D}^{-1} \frac{q^{-\nu \varrho(\alpha)}}{1-q^{\nu-\alpha}} \bar{T}_{\rho, w}^{\prime},
$$

where $\bar{T}_{\rho, w}^{\prime}=\sup _{\|\xi\|_{r}=1} T_{\rho, w}^{\prime}(\xi)$ for the system (2) with $\rho=q^{1+\varrho(\alpha)}$ and $w \in(0,1)$.

If in the conditions of the latter theorem $\nu<0$, then the system is fixed-time stable for $d=0$ as in the case of Theorem 1 (for $\nu=0$ the system is exponentially converging to the origin for $d=0$ from the unit sphere $S_{r}$ ), and in general Theorem 2 is a generalization of Theorem 1. However, the supervision provided in Theorem 2 is qualitatively different from the case of Theorem 1 if $\alpha>0$, since in this case $\mu_{i}$ forms an increasing sequence for $i=\overline{0, i^{*}-1}$.

Example 2. Let us continue to consider Example 1, but for $\nu=0$, then for a linear system (2) we obtain:

$$
a_{1}=1.618, a_{2}=0.138, \gamma(s)=5.854 s \text {. }
$$

Take $D=1$ and

$$
d(t)=D \sin (t)
$$

for simulation. Select $q=1.7, \alpha=0.125$ and $\theta=0.1$, then for $\mu_{D}=10$ all conditions of Theorem 2 are satisfied and the closed loop hybrid system (4), (7), (9) has a finite time of convergence to the unit ball. An example of the system trajectories is given in Fig. 2.

\section{Finite-time convergence to the origin for $\nu \geq 0$}

Let us consider the problem of finite-time convergence to the origin from the unit sphere $\left(z(0) \in S_{r}\right)$ by switching among the systems having asymptotic rate of convergence, i.e. with $\nu \geq 0$. For this purpose the following supervision algorithm will be used for $i \geq 0$ :

$$
\begin{aligned}
t_{i+1} & =\min _{t \geq t_{i}:\left\|M_{i} z(t)\right\|_{r} \leq q^{-1-i}} t, t_{0}=0 \\
\mu_{i} & =\mu_{D} q^{i \alpha}
\end{aligned}
$$

where $q>a_{1}$ and $\alpha>\nu$ are tuning parameters, $\mu_{D}>\max \left\{1, \frac{D}{\gamma^{-1}(1)}\right\}^{1 / n}$ comes from Claim 2 . It is necessary to show that the solutions of (5), (6) are well defined for the supervisory algorithm (10), (11) and that the closed loop system converges 


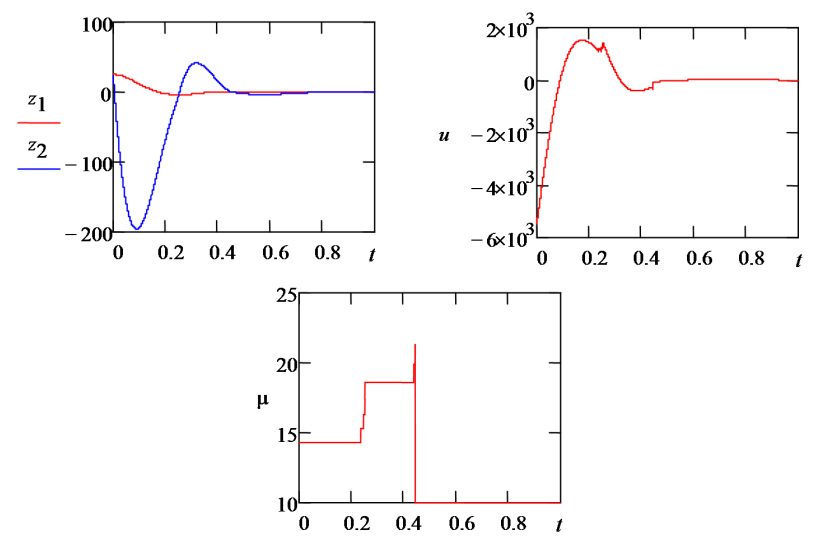

Figure 2. Fixed-time convergence to $B_{r}(1)$ for $\nu=0$ with increasing $\mu_{i}$

to the origin in a finite-time time.

Remark 4. The difference here with respect to the cases considered previously is that there is an infinite number of switches and that asymptotically the system approaches a Zeno behavior or a sliding mode [19], [10]. In practice the switching may be stopped after reaching a certain vicinity around the origin, i.e. when $\left\|z\left(t_{i}\right) \mid\right\|_{r} \leq \varepsilon$ for a given $\varepsilon \in(0,1)$, which is specifying the available precision of computation, for example. In this case there is a finite number $i^{*}=c e i l\left[\ln _{q} \varepsilon\right]$ of switches as previously.

From (11) we obtain for all $i \geq 0$ :

$$
\begin{gathered}
M_{i+1} M_{i}^{-1}=\operatorname{diag}\left\{\left(\frac{\mu_{i+1}}{\mu_{i}}\right)^{1-k}\right\}_{k=1}^{n} \\
=\operatorname{diag}\left\{q^{\alpha(1-k)}\right\}_{k=1}^{n},
\end{gathered}
$$

then ( $\alpha$ and $\nu$ are positive)

$$
\begin{gathered}
q^{\frac{-\alpha(n-1)}{1+(n-1) \nu}-1-i}=q^{\frac{-\alpha(n-1)}{1+(n-1) \nu}}\left\|x\left(t_{i+1}\right)\right\|_{r} \\
\leq\left\|x\left(t_{i+1}^{+}\right)\right\|_{r} \\
\leq\left\|x\left(t_{i+1}\right)\right\|_{r}=q^{-1-i} .
\end{gathered}
$$

The instant $t_{i+1}$ will be generated by (10) when $\left\|x\left(t_{i+1}\right)\right\|_{r}=q^{-1-i}$ and it is well defined (and separated from $t_{i}$ ) if $\frac{\alpha(n-1)}{1+(n-1) \nu}<1$, or equivalently $\alpha<\nu+\frac{1}{n-1}$.

Note that on the interval $\left[t_{i}, t_{i+1}\right)$ for any $i \geq 0$ the control

$$
u(t)=\mu_{i}^{n} K\left(M_{i} z(t)\right)
$$

is a continuous function of time. Due to homogeneity of the control we have:

$$
\begin{gathered}
u(t)=\mu_{i}^{n} K\left(M_{i} z(t)\right)=\mu_{i}^{n} K(x(t))=\mu_{D}^{n} q^{i n \alpha} K(x(t)) \\
=\mu_{D}^{n} q^{i n \alpha}\|x(t)\|_{r}^{1+n \nu} K(\xi(t)),
\end{gathered}
$$

where $\xi(t) \in S_{r}$ is such that $x(t)=\Lambda_{r}\left(\|x(t)\|_{r}\right) \xi(t)$. As it has been shown previously, the amplitude of the state is not growing during the jumps, i.e. $\left\|x\left(t_{i}^{+}\right)\right\|_{r} \leq\left\|x\left(t_{i}\right)\right\|_{r}=q^{-i}$, then

$$
\left\|u\left(t_{i}\right)\right\| \leq \mu_{D}^{n} q^{i n \alpha} q^{-(1+n \nu) i}\|K(\xi)\| \leq \mu_{D}^{n} q^{i[(\alpha-\nu) n-1]} \kappa
$$

with a finite constant $\kappa=\sup _{\xi \in S_{r}}\|K(\xi)\|$, and the control is not growing during the jumps if $(\alpha-\nu) n-1 \leq 0$ or, 
equivalently, if $\alpha \leq \nu+n^{-1}$, and this condition includes the previous one, $\alpha<\nu+\frac{1}{n-1}$, for separation of instants of time $t_{i}$ with $i \geq 0$.

On the interval of time $\left[t_{i}, t_{i+1}\right)$ the dynamics of $x(t)$ is governed by (5) for $\mu_{i}=\mu_{D} q^{i \alpha}$. On this interval $\left[t_{i}, t_{i+1}\right)$ the state $x(t)$ has to decrease in $q$ times. If $\gamma\left(\mu_{i}^{-n} D\right)<q^{-1-i}$, then the relative function of contraction in $q>1$ times with some $w \in(0,1)$ for (2), $T_{q, w}^{\prime}\left(x\left(t_{i}\right)\right)$, can be used to evaluate the length of the interval $\left[t_{i}, t_{i+1}\right)$. From (11), if $\mu_{D}>\max \left\{1, \frac{\vartheta D}{\gamma^{-1}\left(q^{-1}\right)}\right\}^{1 / n}$ for some $\vartheta \geq 1$ we obtain a restriction:

$$
\gamma\left(\mu_{i}^{-n} D\right)=\gamma\left(\mu_{D}^{-n} q^{-i n \alpha} D\right) \leq \gamma\left(\vartheta^{-1} q^{-i n \alpha} \gamma^{-1}\left(q^{-1}\right)\right)<q^{-1-i}
$$

which has to be satisfied for the function $\gamma$ and selected $\vartheta, \alpha$, then

$$
\begin{gathered}
t_{i+1}-t_{i}=\mu_{i}^{-1} T_{q, w}^{\prime}\left(x\left(t_{i}\right)\right) \\
\leq \mu_{D}^{-1} q^{i(\nu-\alpha)} \bar{T}_{q, w}^{\prime},
\end{gathered}
$$

where $\bar{T}_{q, w}^{\prime}=\sup _{\|\xi\|_{r}=1} T_{q, w}^{\prime}(\xi)$, and the interval length $t_{i+1}-t_{i}$ is decreasing with $i$. Finally, the time of convergence to the origin $t_{0}$ can be estimated as follows

$$
\begin{aligned}
t_{0} & =\sum_{i \geq 0}\left(t_{i+1}-t_{i}\right) \\
& \leq \mu_{D}^{-1} \sum_{i \geq 0} q^{i(\nu-\alpha)} \bar{T}_{q, w}^{\prime} \\
& =\mu_{D}^{-1} \bar{T}_{q, w}^{\prime} \sum_{i \geq 0} q^{i(\nu-\alpha)} \\
& \leq \frac{\mu_{D}^{-1}}{1-q^{\nu-\alpha}} \bar{T}_{q, w}^{\prime}
\end{aligned}
$$

since $q^{\nu-\alpha}<1$. Therefore, the time of convergence to the origin from the unit sphere $S_{r}$ in the system (5), (6), (10), (11) is finite and independent in initial conditions. The following result has been proven.

Theorem 3. Let for the system (4) with $\nu \geq 0$ and $d \in \mathcal{L}_{D}$ the supervisory algorithm be selected as in (10), (11) with $q>a_{1}, \mu_{D}>\max \left\{1, \frac{\vartheta D}{\gamma^{-1}\left(q^{-1}\right)}\right\}^{1 / n}, \vartheta \geq 1$ and $\alpha \in\left(\nu, \nu+\frac{1}{n}\right]$ such that

$$
\gamma\left(\vartheta^{-1} q^{-i n \alpha} \gamma^{-1}\left(q^{-1}\right)\right)<q^{-1-i} \quad \forall i \geq 0,
$$

then the solutions of the closed-loop system are well defined for all $t \geq 0$ and for any $z(0) \in S_{r}$ the uniform time of convergence to the origin is less than

$$
\frac{\mu_{D}^{-1}}{1-q^{\nu-\alpha}} \bar{T}_{q, w}^{\prime}
$$

where $\bar{T}_{q, w}^{\prime}=\sup _{\|\xi\|_{r}=1} T_{q, w}^{\prime}(\xi)$ for the system (2) with some $w \in(0,1)$.

The condition (12) can be rather restrictive, it ensures that "Achilles is able to catch Tortoise". For example, in the linear case, if $\nu=0$ and $\gamma(s)=\gamma_{0} s$ for some $\gamma_{0}>0$, then (12) can be reduced to $\vartheta^{-1} q^{i}<q^{i n \alpha}$, which is true for $\alpha \geq \frac{1}{n}$ and $\vartheta>1$ that always has a nonempty intersection with the condition $\alpha \in\left(\nu, \nu+\frac{1}{n}\right]$ for $\nu \geq 0$.

Example 3. Continuing with the same example for $n=2$ and $\nu=0$, with

$$
a_{1}=1.618, a_{2}=0.138, \gamma(s)=5.854 s
$$

$D=1$ and $d(t)=D \sin (t)$ for simulation. Select $q=1.7, \vartheta=1.5$ and $\alpha=0.5$, then for $\mu_{D}=4$, we can observe that all conditions of Theorem 3 are satisfied and the closed loop hybrid system (4), (10), (11) is finite-time converging to the origin from the unit sphere. An example of the system trajectories is given in Fig. 3, where the switching has been stopped when the precision $\|z(t)\|_{r} \leq 5 \times 10^{-5}$ has been reached (the Euler method with the step $10^{-4}$ was used for simulation). 


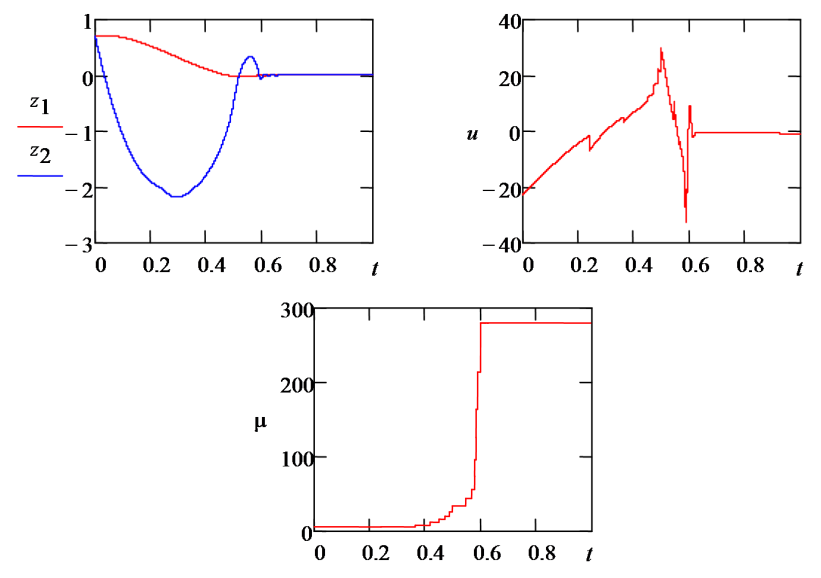

Figure 3. Finite-time convergence from $S_{r}$ to the origin for $\nu=0$ with increasing $\mu_{i}$

\section{CONCLUSION}

The problem of convergence rate acceleration in homogeneous systems by switching among different values of parameters is addressed in this work. The presence of bounded matched disturbances is taken into account. Different algorithms are proposed, which can be applied to homogeneous systems with all signs of degree (negative, zero, positive), and ensuring acceleration of convergence from infinity to the unit sphere or from the sphere to the origin. The obtained results are based on analysis of an auxiliary hybrid system. As a result, taking a linear feedback for linear system it is shown how to scale the gains and switch between them in order to ensure a fixed-time convergence in the closed-loop system. Different strategies are analyzed: decreasing or increasing the gains while approaching the goal set. Note that the obtained results are not related with dwell-time stability since switching stops in a finite time, with interval length between commutations converging to zero in Theorem 3. The results are illustrated by computer simulations for a planar benchmark. Analysis of finite-time and fixed-time stability for hybrid systems can be a direction for future research.

\section{REFERENCES}

[1] I.M. Ananyevskii. Limited control of a rheonomous mechanical system under uncertainty. Journal of Applied Mathematics and Mechanics, 65(5):785796, 2001.

[2] I.M. Ananyevskii. Control synthesis for linear systems by methods of stability theory of motion. Difierential Equations, 39(1):1-10, 2003.

[3] A. Bacciotti and L. Rosier. Lyapunov Functions and Stability in Control Theory. Springer, Berlin, 2nd edition, 2005.

[4] E. Bernuau, A. Polyakov, D. Efimov, and W. Perruquetti. Verification of ISS, iISS and IOSS properties applying weighted homogeneity. Systems \& Control Letters, 62:1159-1167, 2013.

[5] S. P. Bhat and D. S. Bernstein. Geometric homogeneity with applications to finite-time stability. Mathematics of Control, Signals and Systems, 17:101-127, 2005.

[6] Y. Dvir and A. Levant. Accelerated twisting algorithm. Automatic Control, IEEE Transactions on, 60(10):2803-2807, Oct 2015.

[7] Y. Dvir and A. Levant. Sliding mode order and accuracy in sliding mode adaptation and convergence acceleration. In Xinghuo Yu and Mehmet Önder Efe, editors, Recent Advances in Sliding Modes: From Control to Intelligent Mechatronics, volume 24 of Studies in Systems, Decision and Control, pages 129-153. Springer International Publishing, 2015.

[8] C. Edwards and S. Spurgeon. Sliding mode control: theory and applications. Taylor and Francis, 1998.

[9] D. Efimov, A. Levant, A. Polyakov, and W. Perruquetti. On acceleration of asymptotically stable homogeneous systems. In Proc. IEEE CDC, 2016.

[10] L. Fridman. Sliding Mode Enforcement after 1990: Main Results and Some Open Problems, volume 412 of LNCIS, pages 3-57. Springer - Verlag, Berlin Heidelberg, 2011.

[11] H. K. Khalil. Nonlinear Systems. NJ 07458. Prentice-Hall, Upper Saddle River, 1996.

[12] A. Levant. Homogeneity approach to high-order sliding mode design. Automatica, 41(5):823-830, 2005.

[13] Yuandan Lin, Eduardo D Sontag, and Yuan Wang. A smooth converse lyapunov theorem for robust stability. SIAM Journal on Control and Optimization, 34(1):124-160, 1996.

[14] J. Moreno and A. Osorio. Strict lyapunov functions for the super-twisting algorithm. IEEE Transactions on Automatic Control, 57(4):1035-1040, 2012.

[15] E. Moulay and W. Perruquetti. Finite time stability conditions for non autonomous continuous systems. International Journal of Control, 81(5):797803, 2008. 
[16] A. Polyakov. Nonlinear feedback design for fixed-time stabilization of linear control systems. IEEE Transactions on Automatic Control, 57(8):21062110, 2012.

[17] A. Poznyak, L. Fridman, and F. J. Bejarano. Mini-max integral sliding-mode control for multimodel linear uncertain systems. IEEE Transactions on Automatic Control, 49(1):97-102, 2004.

[18] E. Roxin. On finite stability in control systems. Rendiconti del Circolo Matematico di Palermo, 15:273-283, 1966.

[19] V. I. Utkin. Sliding Modes in Control Optimization. Springer-Verlag, Berlin, 1992.

[20] V.I. Zubov. On systems of ordinary differential equations with generalized homogenous right-hand sides. Izvestia vuzov. Mathematica., 1:80-88, 1958 (in Russian). 